\title{
Erythematous plaques of the vulvo-perineal region
}

\author{
Qin Jian Low, Tzyy Huei Lim, Shu Ann Hon, Seng Wee Cheo, Noranizah Wagino, Evelyn \\ Wen Lee Yap
}

Low QJ, Lim TH, Hon SA, et al. Erythematous plaques of the vulvo-perineal region. Malays Fam Physician. 2021;16(3);101-103.

https://doi.org/10.51866/cr1276

\section{Keywords:}

Melkersson-rosenthal

syndrome, orofacial

granulomatosis,

neurocutaneous disorder

\section{Authors:}

\begin{abstract}
Low Qin Jian
(Corresponding author)

MD (UMS), MRCP (UK)

Department of Internal Medicine

Hospital Sultanah Nora Ismail

Batu Pahat, Johor, Malaysia

Email: lowqinjian@moh.gov.my
\end{abstract}

\section{Lim Tzyy Huei}

MD II.M. Sechenov Moscow Medical

Academy), MRCP (UK)

Department of Internal Medicine,

Hospital Sultanah Nora Ismail

Batu Pahat, Johor, Malaysia

\section{Hon Shu Ann}

MBBS (MBBS)

Department of Internal Medicine

Hospital Sultanah Nora Ismail

Batu Pahat, Johor, Malaysia

\section{Cheo Seng Wee}

MD (UMS), MRCP (UK)

Department of Internal Medicine

Hospital Lahad Datu, Lahad Datu

Sabah, Malaysia

\section{Noranizah Binti Wagino}

MBBS (UM), Master of pathology

(Anatomic Pathology)

Department of Anatomical Pathology

Hospital Sultanah Fatimah Muar

Johor, Malaysia

\begin{abstract}
Skin conditions are a common reason for consulting primary health care. The public frequently expects their primary health care providers to be able to recognise and treat common skin conditions with confidence. Primary care providers must be able to identify 'red flag' skin conditions that require dermatology referral. However, diagnosis is often delayed due to the non-specific clinical presentation, low incidence, slow evolution and long-term history of such lesions. We report the case of a patient with extramammary Paget's disease that presented to her primary care provider and subsequently underwent a wide local excision.
\end{abstract}

\section{Introduction}

Extramammary Paget's disease (EMPD) can initially present with chronic eczema-like skin changes around the genital region in both genders. ${ }^{1}$ EMPD is a rare condition with only a few hundred cases reported worldwide. ${ }^{1,2}$ However, the exact incidence remains unknown. EMPD represents approximately $6 \%$ of all cutaneous Paget's disease cases and predominantly affects patients between the ages of 60 and 80 years, with a peak age of 65 years. ${ }^{1}$ It often affects areas where the apocrine sweat glands are abundant. These can occur around the vulva, perianal region, scrotum, penis or axilla. ${ }^{2}$ Frequently, females between the ages of 50 and 60 years are affected and some of the patients may have an underlying malignancy. It is important for primary care providers to identify this classical skin condition early and promptly refer affected patients to a dermatologist for a skin biopsy. The management of EMPD includes surgery and topical therapy. Prognosis depends on the early diagnosis and stage of the disease.

\section{Case presentation}

An 80-year-old woman experienced bilateral pruritic excoriation over her vulva region over a 2-year period. Her underlying comorbidities included hypertension, type II diabetes mellitus and dyslipidaemia. She was initially treated as having vulval candidiasis by her primary care doctor; however, the 'rash' did not improve with the topical therapy prescribed. Local examination revealed an extensive well-demarcated erythematous plaque over her bilateral labia majora extending to her buttocks (Figure A-B). The lesion was confined to her vulval skin. Perspeculum examination was normal. There was no inguinal lymphadenopathy or abdominal mass. Skin biopsy was consistent with EMPD involving her vulva (Figure C-G). She was also screened for synchronous cancer. Her mammogram, pap smear, colonoscopy, cystoscopy and abdominal scans were all unremarkable. She was subsequently referred to the plastic surgery team for a wide local excision of the lesion.

\section{Discussion}

EMPD is an uncommon intraepithelial adenocarcinoma that can affect the anogenital or axillae skin. ${ }^{1}$ The most commonly affected age group is the elderly (over 50 years old). There are two forms of Paget's disease. Primary EMPD of the skin originates from the cutaneous layer, whilst secondary EMPD is associated with adenocarcinoma. Although the aetiology of EMPD remains unclear, the apocrine-rich glands of the skin are commonly involved. The vulva is the most commonly affected site, reported in 65\% of all cases. ${ }^{2}$ Immunohistochemistry can be used to differentiate between primary and secondary EMPD. Cytokeratin 20 will be positive in secondary EMPD but negative in primary EMPD. On the other hand, GCDFP-15 will be positive in primary EMPD but negative in secondary EMPD. GCDFP-15 staining was not performed since the stain was not available. 
CASE REPORT

\section{Evelyn Yap Wen Lee} MBBS (IMU), MRCP (UK) Adv M Derm (UKM)

Department of Dermatology, Hospital Pakar Sultanah Fatimah Muar, Johor Malaysia

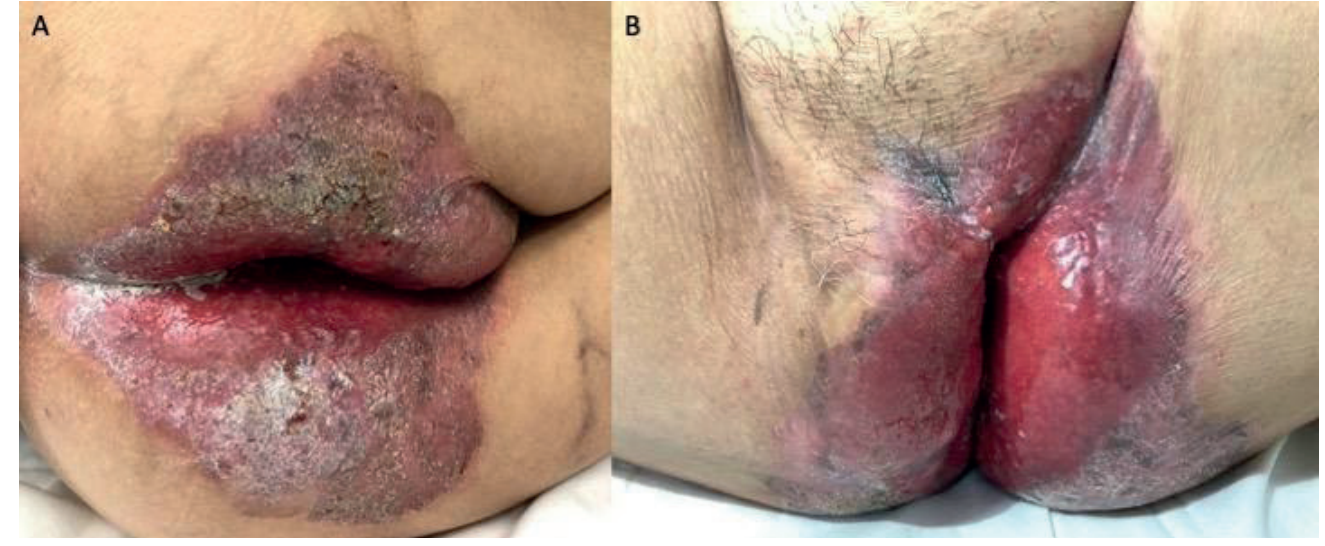

Figure A-B. Well demarcated erythematous plaques seen over the vulval and perianal region.

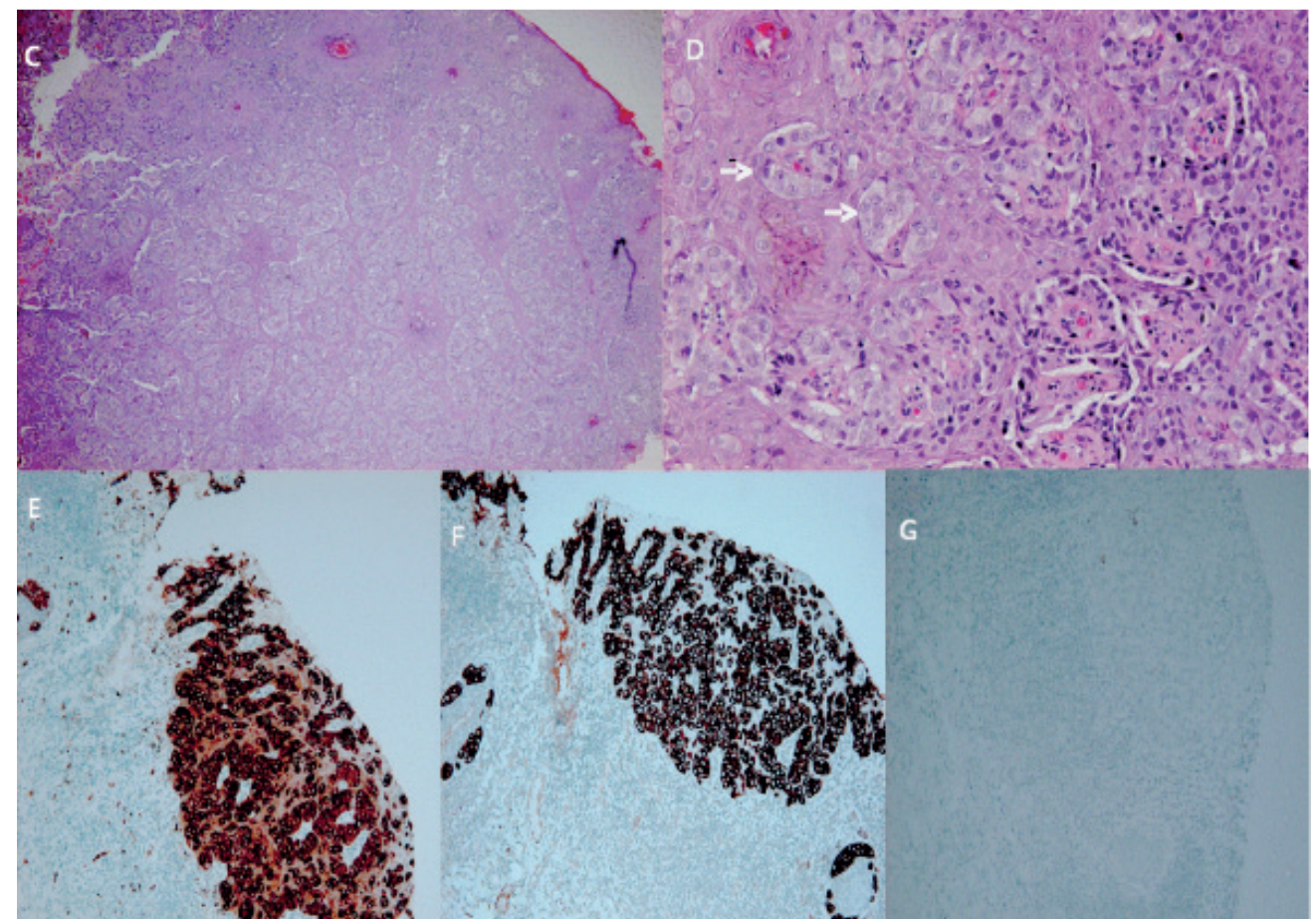

Figure C. Extramammary Paget's disease $(\mathrm{H}+\mathrm{E}$ staining x100). Large, pale malignant cells arranged in clusters and single cells throughout the epidermis. There was no dermal infiltration.

Figure D. (H+E staining x200). Large tumour cells with pleomorphic, oval-shaped vesicular nuclei and abundant pale cytoplasm (white arrows).

Figure E. Paget's cells intensely positive for carcinoembryonic (CEA) antigen.

Figure F. Cytokeratin 7 (CK7) demonstrates the immunoreactivity of tumour cells.

Figure G. Negative cytokeratin 20 (CK 20) immunoreactivity.

Pruritus is commonly reported among patients with vulval Paget's disease, with some potentially experiencing excoriations and lichenification.1 Other reported symptoms include burning pain, irritation and weepy erosions. Due to the persistent pruritic eczematous lesions, many patients have been treated with a course of antieczema therapy. The possibility of synchronous malignancies should be considered since approximately $30 \%$ of these patients have a non-contiguous carcinoma (e.g., involving the breast, rectum, bladder, urethra, cervix or ovaries).
The standard treatment for EMPD includes wide local excision and Mohs micrographic surgery. $^{3}$ Mohs micrographic surgery results in a lower relapse rate when compared to wide local excision. In cases where Paget cells have extended into the reticular dermis or are observed within lymphatics, a sentinel lymph node biopsy is required to decide on the extent of surgery. ${ }^{4}$ In non-surgical candidates, imiquimod cream can be used. In selected cases, radiotherapy may be attempted after a multidisciplinary team discussion. Generally, the 5 -year overall survival rate is $75-95 \%$. Although 
Paget's disease is often intraepithelial, it can progress to the invasive stage with distant metastases in some cases. The diagnosis of EMPD often triggers an extensive age-specific malignancy screening. Patients with EMPD need to have a long-term follow-up since they can develop recurrence. ${ }^{5}$

\section{Conclusion}

Primary care providers should be familiar with the presentation of EMPD and begin a detailed systemic and physical examination to look for any undiagnosed malignancy. Early referral to a dermatologist would be imperative for any patient with chronic nonresolving skin lesions in the anogenital region. Primary care providers must be aware of this rare skin disease (rather than familiar with it) and refer any patient with a non-resolving skin lesion early to rule out malignancy.

\section{Acknowledgements}

The authors would like to thank the DirectorGeneral of Health Malaysia for his permission to publish this article.

\section{Conflicts of interest and funding}

The authors declare that they have no competing interests in the publication of this paper. The authors received no financial support for the research, authorship or publication of this article.

\section{Informed consent}

Written informed consent for this paper (including images, case history and data) were obtained from the patient for the publication of this paper, including the accompanying figures.

\section{References}

1. Extramammary Paget disease: A review of associated underlying malignancies. J Am Acad Dermatol. 2010;62(3):Supplement 1, AB5. doi: http://doi.org/10.1016/j. jaad.2009.11.056.

2. Yildiz P, Ronen S, Aung PP, et al. Extramammary Paget disease-A challenging case. Am J Dermatopathol. 2019;41(11):867868
3. Fanning J, Lambert HC, Hale TM, et al. Paget's disease of the vulva: Prevalence of associated vulvar adenocarcinoma, invasive Paget's disease, and recurrence after surgical excision. Am J Obstet Gynecol. 1999;180(1 Pt 1):24-7.

4. Jones IS, Crandon A, Sanday K. Paget's disease of the vulva: Diagnosis and follow-up key to management; a retrospective study of 50 cases from Queensland. Gynecol Oncol. 2011;122:42.
5. Ito T, Kaku-Ito Y, Furue M (2018): The diagnosis and management of extramammary Paget's disease. Expert Rev Anticancer Ther. 2018 Jun; 18(6): 543-553. doi: 10.1080/14737140.2018.1457955. 